in no more than 60 per cent. of cases on the average. That alone is sufficient reason for using digitalis in the decompensated fibrillators, rather than quinidin.

Dr. Alexander Lambert, New York: I have tried quinidin in thirty cases. In one patient, the fibrillation stopped and the rate fell to normal. In all the others, with careful electrocardiographic control, there was no effect.

Dr. L. F. Bishop, New York: Dr. Hamburger has probably made the best observations and written the best literature on quinidin. So that, in discussing his paper, I do not wish to add to the experimental side of it, but rather to give my own impressions founded on a rather unusual group of patients. For a number of years, I have been using the electrocardiograph in private practice, and I have instructed my patients to come to the office when they have palpitation and have a picture taken of the heart beat. Thus, I have accumulated a number of examples of temporary fibrillation which did not recur again for a long time or did not recur at all. It seems to me that a very important element in appraising the value of quinidin is a knowledge of the natural history of fibrillation. I believe that nearly every example of fibrillation, except in the very advanced broken-down heart, begin in this way. The vast number of fibrillators commence by short attacks which become more frequent and last longer. It is almost a joke on medical science that the best example of therapeutic effect should be disturbed by the introduction of a new treatment. There is no treatment in all modicine as brilliant as the treatment of fibrillation of the auricle with digitalis, and I would say as a matter of experience that the patients do not feel any better when they are treated by quinidin than when they are treated by digitalis. In other words, the chronic fibrillator carries fibrillation comfortably if he takes enough digitalis or if he develops heart block which balances the fibrillation. I have seen persons who have continued very well with fibrillation for fifteen years or more. There is no doubt that there often exist clots in the paralyzed auricle in fibrillation, and it is cerainly dangerous to restore the contraction of that auricle if it is going to throw out an emloolus which may kill the patient. I do not believe that quinidin is a valuable general heart tonic, and I believe that men in general practice will do well if for the present they leave the use of quinidin to men who are experimenting with it in hospitals. I think it is unwise to use it in ambulatory patients in private practice.

Dr. Walter W. Hamburger, Chicago: I agree with Dr. Bishop in the main. I think his warning in the advanced cases should be emphasized. The real danger is, of course. embolism. If there is any sign or history of embolism, quinidin should not be given. One of my patients is a surgeon who has had paroxysmal attacks of fibrillation for fifteen years. He gets attacks once a year or so, sometimes while operating; once while swimming, and the last time while working in his garden. The attack last year lasted ahout a week and was associated with severe anginal pains. $\mathrm{He}$ was worried about it and was quite ill. After a week's rest in bed, the fillrillation stopped of its own accord, and he has had no attack until a week ago. I immediately gave him 3 grains $(0.2 \mathrm{gm}$.) of quinidin and another dose in two hours. Within three hours this attack was over: and he is confident the drug shortened the attack. Likewise in the paroxysmal attacks of exophthalmic goiter, quinidin undoubtedly controls the attacks. It should not be given to ambulatory patients, and it should not be given as a routine until we know much more about it than we do at the present time. Embolism occurs in fibrillation without quinidin and sometimes without the estallishment of a normal rhythm.

Purpose of Operation for Epilepsy.-In operating on epileptics, and in choosing cases for operation, we should like to have a clear idea of the purpose of the operation, based on some rcasonable conception of that relationship. The surgeon who merely rcmoves a disk of bone from the cranium of an epileptic paticnt places himself on a level with the practitioner of the stone age, the marks of whose handiwork on the skulls, probably of the epileptic or the insane, are to be seen in museums of ethnology.-Percy Sargent, Brain 44:314 1921.

\section{THE CAUSE AND RELIEF OF ACUTE INTESTINAL OBSTRUCTION *}

\author{
CHARLES H. MAYO, M.D. \\ ROCHESTER, MINN.
}

Ileus is one of the most serious conditions that confront the medical attendant, since if it is not relieved death is inevitable. In order to lower the death rate in such conditions, much depends on early diagnosis, judgment and promptness of action. Relief is by no means possible in all cases; for instance, there is necessarily a high mortality in cases of mesenteric embolism and thrombosis. Therefore, the discussion of the subject of acute intestinal obstruction, from time to time, is of value, not so much for the older practitioners as for those who have more recently entered the profession, so that they may acquire knowledge without encountering the tragedies which fixed the memory of such conditions in the minds of those long in practice.

Cases of acute intestinal obstruction may be regarded as falling naturally into four groups: (1) apparent obstruction, really intestinal stasis, a reflex symptom occurring with slight abdominal distention, the pulse and temperature changes being such as are caused by the primary lesion, which is often renal; (2) the obvious hernia; (3) acute obstruction from an intraabdominal lesion, and (4) postoperative obstruction.

The obvious hernias, inguinal, femoral, umbilical and ventral, which formerly caused a considerable proportion of obstructions through strangulation, have now largely been taken from this group, as even laymen appreciate the safety of modern surgery over that of a few decades ago. Hernia repaired at an elective period prevents the high percentage of strangulations formerly seen, when a reasonable fear of surgery led to prolonged taxis which often aided in the development of gangrene. The wasted opportunity, adcled to the seriousness of preantiseptic surgery, gave a high mortality which resulted in making acceptable this vicious circle of delay. Gangrene, or wet death of tissue, especially abdominal, and when sepsis is possible, furnishes toxins which are most difficult to counteract without the removal of the destroyed tissue or without adequate drainage.

Obstructions within the abdomen are those which cause the greatest apprehension, as there are added difficulties of diagnosis, some of which have been overcome by fluoroscopic observation or roentgenograms revealing obstructions after the opaque material has been given by mouth or by rectum. Volvulus from the twist of an extra long mesentery, usually of the sigmoid, produces not only obstruction but also the toxins of tissue death. Various forms of internal hernia, beneath bands, through congenital or unnatural mesenteric openings or through the diaphragm, usually cause degrees of partial, chrcnic, rather than acute, obstruction, which, however, may suddenly supervene. A second form of toxin concerning which much has been written is still under discussion. It is observed in obstructions high in the jejunum, blocking the duodenum, and, although the exact cause of retention is not always known, the condition is recognized. Such an obstruction around the duodenojejunal juncture, with paresis and dilatation of the duodenum, may caise

* Read before the Section on Obstetrics. Gynecology and Abdominal Surgery at the Seventy. Third Annual Session of the American Medical A sociation, St. Leuis, May, 1922. 
alkalosis with tetany, in which there is loss of the chlorids and a great general loss of calcium which chokes the renal tubules. This is most serious and is attended by vomiting of green fluids having but little odor. At first, the vomitus is usually watery and contains undigested food particles, and later bile; but the green vomitus probably originates largely in the upper duodenum. In obstruction of the jejunum or ileum, the green vomitus may be noted the second day, followed later by regurgitant brown and darker colored fecal or foul smelling fluids. The true toxin of pancreatic and duodenal ferments is most difficult to counteract; but relief is obtained or life is prolonged by removing the fluid at regular and frequent intervals with a stomach tube. The mortality is high because efforts to relieve the obstruction surgically are made late. The operation must drain the duodenum below by jejunostomy and a tube passed to the duodenum, or through the stomach, by gastrostomy and a tube through the py'orus, or by duodenojejunostomy.

Obstruction of the stomach at the pylorus causes death from inanition, yet if fluids can be supplied subcutaneously, by rectal tube or nutritional jejunostomy tube, life can be prolonged for a considerable period. Obstruction below this point becomes serious early because of the toxemia, although danger of this is less acute, the lower down the obstruction occurs; and if the obstruction is in the large bowel, several days may be added to the possible period of surgical relief over that if the obstruction is in the sma'l intestine. In the latter case, the later the operation, the higher the mortality for any given procedure. After the first day each hour increases the danger of the condition. The toxemia then from colonic obstruction is of less relative importance than perforation, peritonitis and inanition, if the stomach is kept empty by regular tubation.

Intussusception, with its sudden onset, cramps and pain, with blood and mucus, may often be located by a palpable tumor, especially in children under 1 year of age, among whom from one third to one half of such conditions are found. In adults intussusception is often started by a tumor, benign or malignant, of the small intestine. The papilloma or adenoma becomes grasped in peristaltic action, the bowel being drawn in behind as it moves forward within its lumen, usually thus producing a tumor of palpable size. An inverted Meckel's diverticulum may act in the same way, or a stiff infected area on the cecum around the base of the appendix may become the invaginating head. Operations are rarely performed early enough that the intussusception may be milked back, and it is usually necessary to perform some degree of resection of the bowel and intestinal anastomosis. Gross tuberculosis in the small or large bowel occasionally causes complete obstruction following a series of chronic, recurring incomplete obstructions. In the large bowel, malignancy accounts for the great majority of these conditions; the obstruction is rarely due to the pressure of tumors except those that are usually obvious or palpable; and even in cancerous obstruction, in most instances, there are recurring attacks of incomplete obstruction which are characterized by the passage of blood streaked mucus before it becomes complete. Sudden, severe pain with shock usually signifies the completion of obstruction, especially if due to a twist or to mesenteric thrombi or emboli, the latter, as a rule, being secondary to endocarditis.

In hernia, gangrene, volvulus or vascular obstruction, there is usually a rapid increase in the leukocyte count. A large gallstone may ulcerate through the gallbladder and duodenal walls, and, after passing through the upper intestine, cause a block in the lower ileum. While a previous history of probable gallstone disease may be elicited after operation, indication for exploration in acute ileus will continue, and the procedure will disclose many causes of obstruction that are relievable and some that are unrelievable. Promptness of action is essential in making an exact diagnosis of the cause.

In many years of active surgical work I have not seen death occur as the result of an unnecessary exploration. I have seen many patients saved and some die of disease, but many died solely because exploration was undertaken too late.

\section{TREATMENT}

Most patients are moribund or nearly so at the time procedures are undertaken for the relief of obstruction of the large bowel. If the obstruction is the essential feature, I should warn against any endeavor to make the operation other than a life-saving drainage procedure, as colostomy, enterostomy or appendicostomy. The last is of little value and becomes absolutely dangerous, unless the appendix is large and patulous and will admit the passage of a large catheter without tension; otherwise cecostomy is indicated. 
Exploration must be made to deternine whether or not the obstruction is malignant or is due to congenital diverticulitis. Sometimes this is impossible because of adhesions and infections. Acquired diverticulitis is rarely so small in area or confined to so few or to such small pockets that the diverticula will not show in the bariun shadow. True congenital diverticula are more rare, resemble malignancy and may even develop malignancy. If the condition is malignant, examination of the liver, glands and intestines should be made in order to ascertain whether future operation is advisable and just what procedure should be carried out.

An endeavor to make the primary operation complete, with removal of the growth, probably doullles the danger over that of a two-stage procedure.

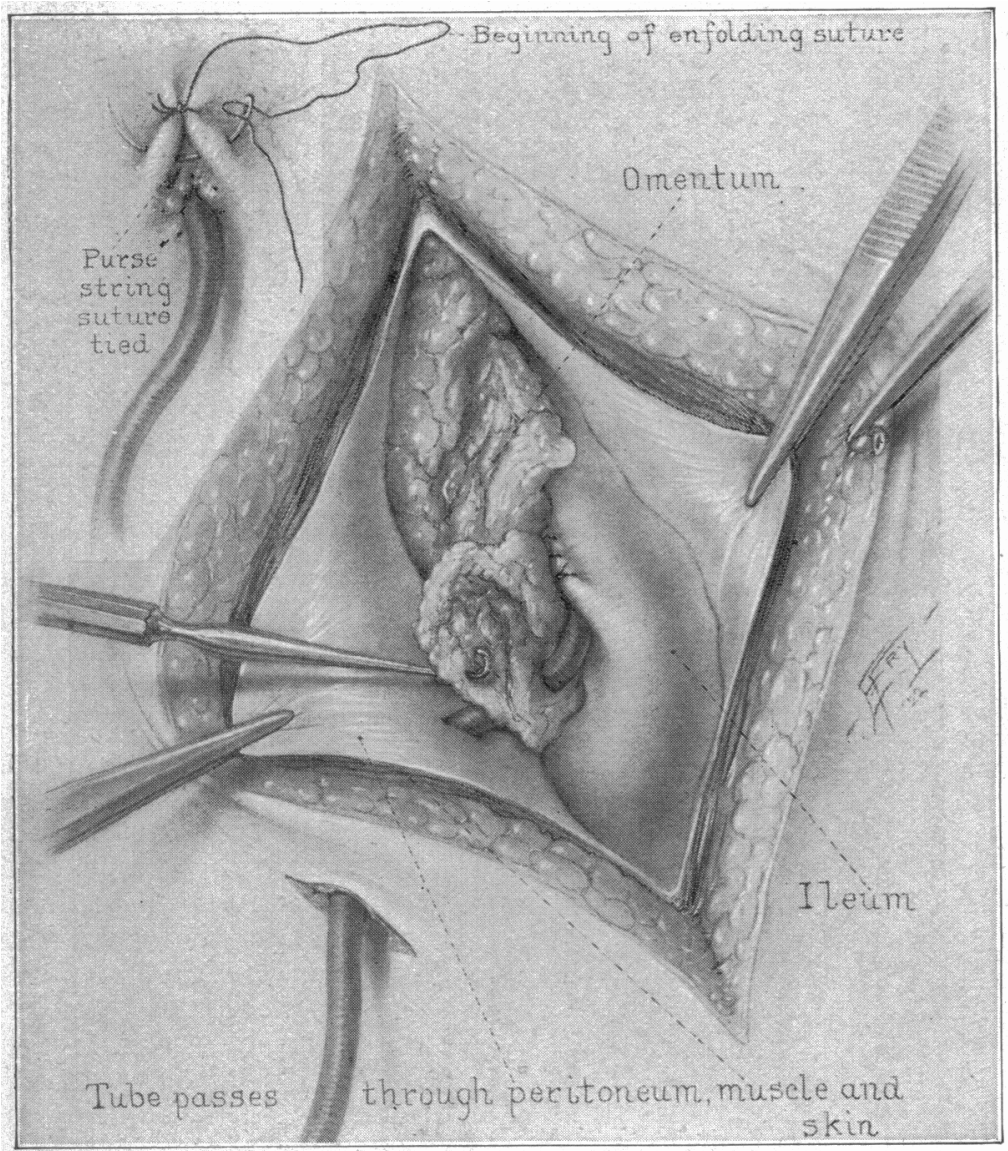

Fig. 2-Abdominal wound showing position of rubber tube in relation to the jleum in its course as it passes through the cmentum and abdominal wall,

Obstruction following operative procedures such as gastro-enterostomy, or the removal of pelvic infections is a real tragedy. While we blame the patient and the medical attendant for delay in calling council in the obscure cases of obstruction, the same delay occurs in hospitals in postoperative cases, and there is a lack of promptness of action, because of the hope that the obstruction is partial and will "clear up tomorrow."

In diagnosing localized peritonitis with the stethoscope, one may hear some tinkling movement of fluids and gases which is not noted in general peritonitis with attendant obstruction from paresis. General peritonitis is accompanied by an even distention, as compared with the irregular distention accompanying peristaltic activity of the stomach and small intestines, which is more manifest the lower the obstruction.
When complete obstruction supervenes, several small, loose, natural, but painful, movements may occur, and there is violent peristaltic action above in the endeavor first to overcome the obstruction, and failing in this to reverse peristalsis and empty the bowel contents into the stomach. Usually vomiting and rarely dilatation occurs. The latter doubles the danger if unrecognized. Therefore, the use of the stomach tube is the most valuable test we have for the purpose of determining the condition of the stomach and the amount of intestinal contents therein, and it must be used repeatedly to prevent dilatation. I should prefer to see a stomach tube rather than a stethoscope hanging around the neck of my surgical intern.

If local obstruction occurs following gastrocnterostomy, turning the patient on his face or on the left side sometimes aids in the passage of gastric contents into the intestine, probably because the opening is on that sicle, or possibly because of a change in circulation of the superior mesenteric vessels. In postoperative obstruction, if the abdomen is opened within twenty-four or thirty-six hours after the beginning of symptoms, the filmy adhesions causing kinks or twists can be separated with little difficulty, and with immediate benefit. Later, but little exploration is advisable for the purpose of ascertaining the cause or exact location of the obstruction, and enterostomy, as advised by Nélaton, should be made for relief. If the obstruction has followed pelvic operation, an ileostomy may afford marked relief; a high obstruction demands jejunostomy for drainage or for nutritional purposes, and if the obstruction follows a gastro-enterostomy, the passage of a catheter into the stomach and down the jejunum through the gastroenterostomy will accomplish the same results without narrowing the lumen of the jejunum. In the presence of tetany, the tube must traverse the duodenum, being passed through the pylorus, and the patient saturated with lime.

Early operation may be performed by reopening the abdominal incision. Operation for obstruction after five days is best performed through a separate incision (Figs. 1 and 2), as the cause of obstruction may be infection in the primary wound, with attachment and kink of the bowel at the infected point, and reopening the incision may spread the infection within the abdomen. This may be prevented by working through a new incision. The enterostomy tube for drainage or nutrition should also be passed through the omentum; and if infection was the cause of the trouble, the tube should be brought through a separate perforation of the abdominal wall at the point of election, permitting a better closure of the working incision. This layer of omentum serves the same purpose as does the cellulose layer in the outer wall of battleships, closing the opening and preventing leakage after removal of the tube. It also gives greater freedom than a direct adhesion of the intestine to the abdominal wall permits. Such operations should be performed as far as possible under local anesthesia, or, in exceptional cases, under combined local and general anesthesia. 
In operations for relief of obstruction, it is often advantageous to be able to distinguish the proximal from the distal end of a loop of bowel. Monk has suggested that the bowel be held in line with the spine, the thumb and finger enclosing the intestine. If they are slipped down the mesentery, and if they pass directly to the mesenteric attachment without being reversed, the upper end is known to be proximal. In order to distinguish the part of intestine to which an individual loop probably belongs without handling a great part of it, one should remember that the jejunum is much thicker and has a larger lumen than the lower ileum; also, the upper, thicker bowel has a thinner mesentery with translucent spaces, and there is much fat in the mesentery of the ileum which becomes thicker nearer its termination.

\section{ABSTRACT OF DISCUSSION}

Dr. George W. CriLe, Cleveland: Dr. Mayo has covered the subject, but $I$ wish to emphasize three points which are most important: 1. Intestinal obstruction demands immediate action, and yet the most careful and gentle action compatible with the relief of the obstruction. It is essential not to do too much. 2. It is extremely important in these cases to build up the water equilibrium of the patient who has been vomiting and has not been absorbing water. Large amounts of water, even 7,000 c.c., subcutaneously, are indicated the first twenty-four hours. 3. In order that the patient may have the proper oxidation within the tissues of the body, it is important, unless the circulation is good, to give a blood transfu. sion, and to give it in advance of the indication of failure of the patient's organism. If necessary, morphin should be prescribed. I am not afraid of giving large doses of morphin after the obstruction is relieved.

Dr. C. H. MAyo, Rochester, Minn.: Twenty-five or thirty years ago appendicitis was discussed at every meeting of the Amcrican Medical Association. Ochsner, Deaver, McBurney, Murphy and others did a great deal to show that operation for appendicitis is safe in the first twenty-four hours, and that the danger is in the second, third and fourth days. Ochsner's method of treating appendicitis, seen too late for a safe early operation, and too early for a safe late operation, saved many hundreds of lives. It appears to me that the mortality from appendicitis is greater today than it was twelve years ago, when we stopped discussing the subject. The important point to teach the younger men in the profession is that no time should be lost in examining such patients and in operating while it is safe to do so, instead of waiting for thirty-six or forty-eight hours. Appendicitis is easily handled if it is attended to early. A patient will never be lost because of a necessary exploration, and many may be saved.

Excessive Accident Fatalities in United States.-The statistical bulletin of the Metropolitan Life Insurance Company announces that the number of deaths from accidents in the United States is twice as high in proportion to the population as that prevailing in England and Wales. In the year 1920 , which was characterized by lower accident rates than any year in the decade, there were 30,000 more deaths than would have been reported if the death rates of England and Wales had prevailed. For the entire country, this means an excess of nearly 37,000 deaths. The subjoined table presents the death rates per hundred thousand from principal extcrnal causes of death, 1920, in the United States registration area and in England and Wales:

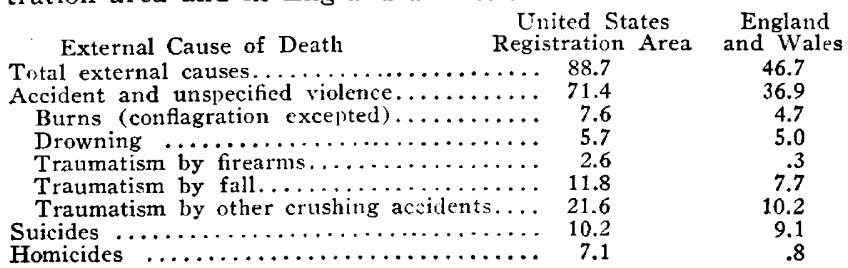

PRIMARY CHANGES IN EYES OF RATS WHICH RESULT FROM DEFICIENCY OF FAT-SOLUBLE A IN DIET*

SHINNOSUKE MORI, M.D.

Chief of the Ophthalmological Clinic, Soutl Manchuria Railway Company Hospital

DAIREN, MANCHURIA

Of recent years, several investigators have described the eye disease which occurs in rats as the result of diets deficient in fat-soluble A. Various names have

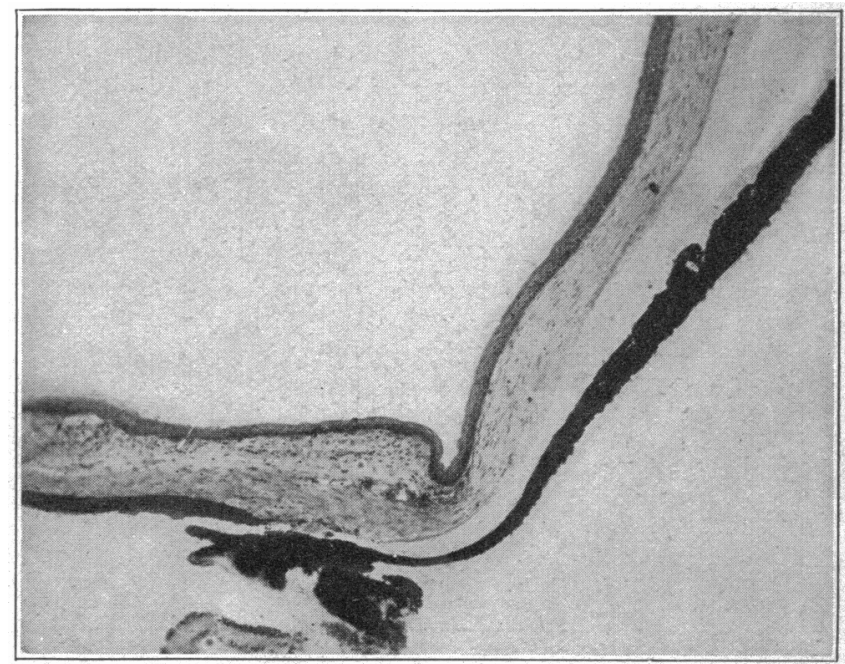

Fig. 1.--Section through the normal eye in the region of the limbus.

been given to this condition. McCollum and Simmonds ${ }^{1}$ termed it xerophthalmia. They chose this name after careful study of the development, symptoms and treat-

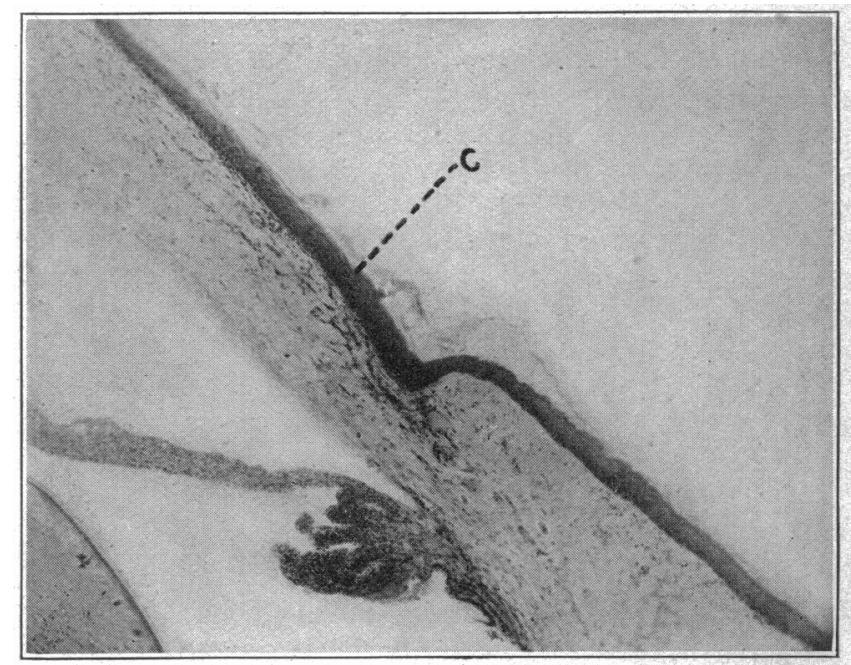

Fig. 2.-Section through cornea and conjunctiva to show the beginning xerosis corneae and conjunctivae, and the cornification $(c)$ and thicken ing of the epithelium. The substantia propria is thickened and infil trated.

ment of xerophthalmia as it occurs in rats and man. Osborne and Mendel ${ }^{2}$ preferred to use the more general term ophthalmia. Goldschmidt ${ }^{3}$ studied the pathology of the disease as it occurred among young

* From the Laboratory of the Department of Chemical Hygiene, School of Hygiene and Public Health, Johns Hopkins University. 1. McCollum, E. V., and Simmonds, Nina: J. Biol. Chem. 32 : 181 ( $N$ (ov.) 1917.

2. Osborne, T. B., and Mendel, L. B.: Ophthalmia and Diet, J. A M. A. 76: 905 (April 2) 1921 .

3. Goldschmidt,. M.: Arch. f. Ophth. 90:345. 1915. 